\title{
ON THE EXTENDED THOMAS-FERMI APPROXIMATION TO THE KINETIC ENERGY DENSITY म
}

\author{
M. BRACK, B.K. JENNINGS and Y.H. CHU \\ Department of Physics, State University of New York, Stony Brook, New York 11794, USA
}

Received 9 September 1976

\begin{abstract}
The extended Thomas-Fermi (ETF) model is used to express the average kinetic energy density $\tilde{\tau}(r)$ of a system of independent particles in terms of the average nucleon density $\tilde{\rho}(r)$. Numerical tests made using Strutinsky-averaged densities from a local Woods-Saxon potential indicated that using terms up to and including the Weizsäcker term is not sufficiently accurate. However, if the next order terms are included, the average kinetic energy of a large nucleus is reproduced to within $2-5 \mathrm{MeV}$. The expansion is not very useful for the exact densities as it leaves out all shell effects. We have also expanded the ETF approximation to include the effects of nonlocalities in the potential; in particular the effective mass and spin-orbit contributions to the kinetic energy are given.
\end{abstract}

The considerable success of recent Hartree-Fock (HF) calculations of nuclear binding and deformation energies $[1,2]$ is largely due to the simplicity of the effective interactions of the Skyrme type $[3,1]$. For these interactions (as well as for more general ones, if the density-matrix expansion [4] is used), the total energy of a finite nucleus can be written in terms of an energy density $\epsilon(r)$ which depends in a simple way on the kinetic energy densities $\tau_{\mathrm{n}, \mathrm{p}}(r)$, the nucleon densities $\rho_{\mathrm{n}, \mathrm{p}}(r)$ and their gradients:

$E_{\text {tot }}=\int \epsilon(r) \mathrm{d}^{3} r=\int \epsilon\left[\tau_{\mathrm{n}}, \tau_{\mathrm{p}}, \rho_{\mathrm{n}}, \rho_{\mathrm{p}}, \nabla \rho_{\mathrm{n}}, \nabla \rho_{\mathrm{p}}, \ldots\right] \mathrm{d}^{3} r$,

$\rho_{\mathrm{n}, \mathrm{p}}(r)=\sum_{i=1}^{N, Z}\left|\phi_{i}^{\mathrm{n}, \mathrm{p}}(r)\right|^{2}, \quad \tau_{\mathrm{n}, \mathrm{p}}(r)=\sum_{i=1}^{N, Z}\left|\nabla \phi_{i}^{\mathrm{n}, \mathrm{p}}(r)\right|^{2}$.

As the constrained HF calculations for heavy deformed nuclei [5] require large amounts of computer time it is of practical interest to find faster ways of obtaining deformation energies from a given effective interaction. It has been shown recently [6] that it is sufficient to solve the self-consistency problem on the average, using statistically smoothed densities $\tilde{\tau}$ and $\tilde{\rho}$, and to add the shell effects perturbatively with Strutinsky's method [7]. In this way one can not only obtain liquid drop like deformation energies microscopically, but also very accurately reproduce the exact $\mathrm{HF}$ results by adding the first order shell-correction.

In order to calculate selfconsistent average binding energies, one may thus use semiclassical models [8]. One essential step is to express the kinetic energy density $\tau(r)$ as a functional of the density $\rho(r)$ in order to avoid the explicit use of single particle wave functions ${ }^{\ddagger 1}$. One way of doing this is to use semiclassical expressions for $\tau(r)$ and $\rho(r)$ in terms of the one body potential $V(r)$ and then to eliminate $V(r)$ to obtain $\tau(r)$ in terms of $\rho(r)$ [9]. The results of this procedure is:

$$
\begin{aligned}
\tau(r) & =\frac{3}{5}\left(3 \pi^{2}\right)^{2 / 3} \rho^{5 / 3}+\frac{1}{3} \nabla^{2} \rho+\frac{1}{36} \frac{(\nabla \rho)^{2}}{\rho}+\frac{1}{4320}\left(3 \pi^{2}\right)^{-2 / 3} \rho^{1 / 3} \\
\times & {\left[24 \frac{\nabla^{4} \rho}{\rho}-60 \frac{\nabla \rho \cdot \nabla^{2} \rho}{\rho^{2}}-28\left(\frac{\nabla^{2} \rho}{\rho}\right)^{2}-14 \frac{\nabla^{2}(\nabla \rho)^{2}}{\rho^{2}}+\frac{280}{3} \frac{(\nabla \rho)^{2} \nabla^{2} \rho}{\rho^{3}}+\frac{184}{3} \frac{\nabla \rho \cdot \nabla(\nabla \rho)^{2}}{\rho^{3}}-96 \frac{(\nabla \rho)^{4}}{\rho^{4}}\right] . }
\end{aligned}
$$

Work supported in parts by USERDA Contract E(11-1)-3001.

$\neq 1$ The subscripts $\mathrm{n}, \mathrm{p}$ will be suppressed in the following; $\tau(r)$ and $\rho(r)$ denote the densities of one kind of nucleus. 
The different contributions $\widetilde{E}_{k}^{\mathrm{TF}}, \widetilde{E}_{k}^{(2)}$ and $\widetilde{E}_{k}^{(4)}$ (see eq. 5) to the total LTF kinetic energy for a system of $N=126$ particles $(A=$ 208 ) in a deformed Woods-Saxon potential [11]. ( $c$ is the larger semiaxis in units of $R_{0}$. .) $\Delta \widetilde{E}_{k}$ is the error made in the total average kinetic energy by using eq. (3) with the averaged density $\rho$ eq. (4). $\Delta E_{k}$ is the corresponding error when the exact density $\rho$ eq. (2) is used. $\delta E$ is the total shell correction.

\begin{tabular}{ccccccr}
\hline$c$ & $\widetilde{E}_{k}^{\mathrm{TF}}$ & $\widetilde{E}_{k}^{(2)}$ & $\widetilde{E}_{k}^{(4)}$ & $\Delta \widetilde{E}_{k}$ & $\Delta E_{k}$ & $\delta E$ \\
\hline 1.0 & 2439.5 & 32.9 & 13.9 & 1.7 & 8.0 & 9.1 \\
1.1 & 2436.9 & 33.0 & 13.9 & 1.6 & 0.0 & 0.3 \\
1.2 & 2434.1 & 33.5 & 14.2 & 1.7 & 1.5 & 1.1 \\
1.3 & 2432.8 & 34.7 & 15.4 & 1.4 & 1.3 & 1.9 \\
1.4 & 2431.7 & 35.9 & 16.6 & 1.8 & 0.4 & -0.1 \\
1.5 & 2421.1 & 37.2 & 17.3 & 2.2 & 2.7 & 2.4 \\
\hline
\end{tabular}

The first term is the Thomas-Fermi (TF) result, the second term vanishes upon integration and the third term is the usual Weizsäcker term (originally derived by Weizsäcker [10] with a different coefficient). The result (3) is only valid for a local, velocity independent one body potential.

Our present purpose is to check numerically the ETF expansion (3) for a realistic local Woods-Saxon potential. Since we cannot expect it to be valid for densities containing shell effects, we use the averaged densities obtained with the Strutinsky occupation numbers $[11] \tilde{n}_{i}$ :

$\tilde{\rho}(r)=\sum_{i}\left|\phi_{i}(r)\right| \tilde{n}_{i}, \quad \tilde{\tau}(r)=\sum_{i}\left|\nabla \phi_{i}(r)\right| \tilde{n}_{i}$.

Inserting $\tilde{\rho}(r)$ into eq. (3) leads to the ETF kinetic energy density $\tilde{\tau}_{\text {ETF }}(r)$ which can be tested against the exact average density $\tilde{\tau}(r)$. However, a comparison of the two functions $\tilde{\tau}(r)$ and $\tilde{\tau}_{\mathrm{ETF}}(r)$ themselves would not make much sense as some of the terms in eq. (3) (e.g. the second one) do not contribute to the total integrated kinetic energy, which we are interested in here. We thus define $\widetilde{E}_{k}$ and $\widetilde{E}_{k}^{\mathrm{ETF}}$ as the total kinetic energies obtained by integrating $\tilde{\tau}(r)$ and $\tilde{\tau}_{\mathrm{ETF}}(r)$, respectively, over $r$ and multiplying with $\hbar^{2} / 2 m$. The latter energy we write as

$\widetilde{E}_{k}^{\mathrm{ETF}}=\widetilde{E}_{k}^{\mathrm{TF}}+\widetilde{E}_{k}^{(2)}+\widetilde{E}_{k}^{(4)}$,

where $\widetilde{E}_{k}^{\mathrm{TF}}$ and $\widetilde{E}_{k}^{(2)}$ are the integrals of the TF and the Weizsäcker terms, respectively. The energy $\widetilde{E}_{k}^{(4)}$ contains the last seven terms of eq. (3) and reduces after partial integrations to the form given by Hodges [9].

In our numerical calculations we used the Woods-Saxon potential described in ref. [11] both for spherical and axially deformed shapes, without Coulomb or spin-orbit terms. Table 1 shows the results obtained for the case of $N=126$ neutrons (in a potential corresponding to $N+Z=A=208$ ) at a series of deformations $c$ which occur typically along the fission path of heavy nuclei. (See ref. [11] for details of the parametrization; $c=1.0$ is the spherical case, $c=1.6$ corresponds to the saddle point deformation in actinide nuclei.) Columns $2-4$ give the separate contributions to $\widetilde{E}_{k}^{\mathrm{ETF}}$, the fifth column shows the difference $\Delta \widetilde{E}_{k}=\widetilde{E}_{k}-\widetilde{E}_{k}^{\mathrm{ETF}}$. We see that the error made in the average kinetic energy using all terms of eq. (3) is less than $2 \mathrm{MeV}$ at moderate deformations and does not increase much even at larger deformations. If only the Weizsäcker term $\left(\widetilde{E}_{k}^{(2)}\right)$ were added to the TF energy an error of $\sim 15-22 \mathrm{MeV}$ would be made which is systematically increasing with deformation.

In order to demonstrate that the expansion (3) is not able to correctly reproduce shell effects, we have included in column 6 of table 1 the difference $\Delta E_{k}=E_{k}-E_{k}^{\mathrm{ETF}}$ obtained in the same way as above but using the exact quantum mechanical densities $\tau(r)$ and $\rho(r)$, eq. (2), together with eq. (3). Even including all terms one makes errors in $E_{k}$ which oscillate strongly with deformation. The oscillations in $\Delta E_{k}$ are clearly correlated to those in the shellcorrection energy $\delta E$, calculated in the usual way $[7,11]$ and displayed in the last column.

Similar results for different nucleon numbers $36 \leqslant N \leqslant 146$ at spherical shape are presented in the figures. In fig. 1 the quantity $\Delta \widetilde{E}_{k}$ is shown as a function of $N$. Again it is small (less than $2 \mathrm{MeV}$ ) and rather smooth. The 


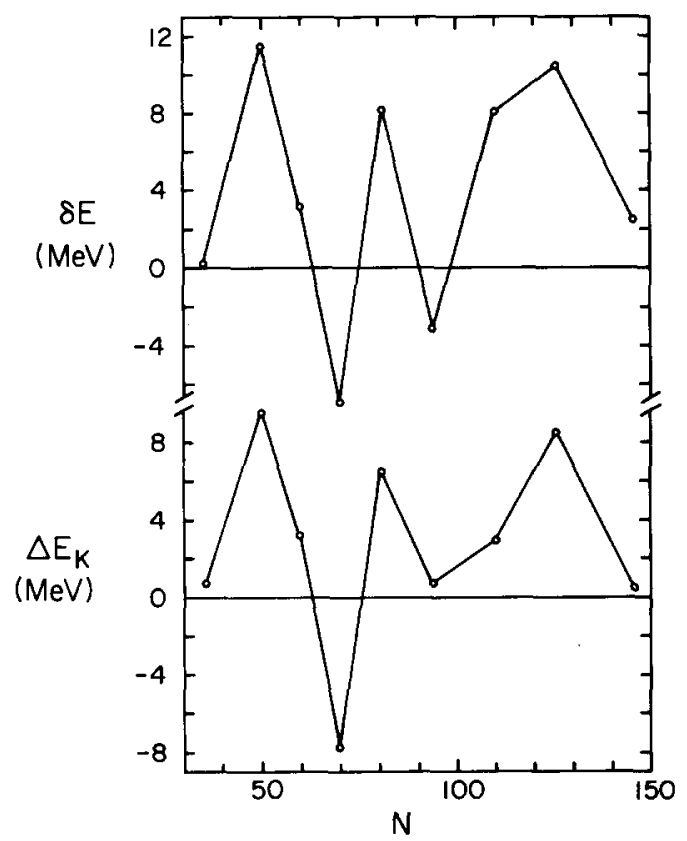

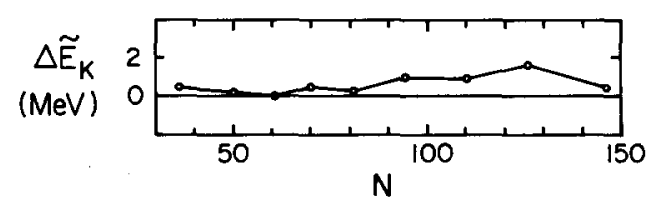

Fig. 1. The error $\Delta \widetilde{E}_{k}=\widetilde{E}_{k}-\widetilde{E}_{k}^{\mathrm{ETF}}$ in the average kinetic energy made by the ETF approximation eq. (3), plotted versus the particle number $N$. (Spherical Woods-Saxon potential.)

difference $\Delta E_{k}$ obtained with the exact densities is shown in fig. 2 (lower curve); as a function of $N$, too, it has strong oscillations which are in close correlation to the shell-correction $\delta E$ (upper curve).

So far we have only discussed a local, velocity independent potential. However, in a realistic calculation one cannot ignore the effective mass and spin-orbit effects. For example, the HF one body Hamiltonian obtained with Skyrme like forces has the form (see e.g. ref. [1])

$H=-\frac{\hbar^{2}}{2 m} \nabla \cdot f(r) \nabla+V(r)+\mathrm{i} S(r) \cdot(\nabla \times \sigma)$,

where $f(r)$ is the ratio of the free nucleon mass $m$ to the effective mass $m *(r), S(r)$ is a spin-orbit form factor and $\sigma$ the Pauli spin matrix. It is no problem in principle to find the semiclassical expansion for the expectation value of the Hamiltonian (6). For the spin--orbit part this has already been done [12].

Whereas it would be an extremely lengthy procedure to work out the expansion of $\tau(r)$ to the same order as in eq. (3) in this general case, it may easily be worked out to the same order as the Weizsäcker term. Proceeding in the same way as in obtaining eq. (3), we get

$$
\tau_{\mathrm{ETF}}(r)=\left(3 \pi^{2}\right)^{2 / 3} \frac{3}{5} \rho^{5 / 3}+\frac{1}{3} \nabla^{2} \rho+\frac{1}{36} \frac{(\nabla \rho)^{2}}{\rho}+\frac{1}{6} \frac{\nabla f \cdot \nabla \rho}{f}-\frac{1}{12} \rho \frac{(\nabla f)^{2}}{f^{2}}+\frac{1}{6} \frac{\nabla^{2} f}{f}+\left(\frac{2 m}{\hbar^{2}}\right)^{2} \frac{1}{2} \rho \frac{(S)^{2}}{f^{2}} .
$$

Similarly, one gets for the spin-orbit energy densities (see e.g. ref. [1]) in lowest order

$$
J_{\mathrm{ETF}}(r)=-\frac{2 m}{\hbar^{2}} \boldsymbol{S}(r) \frac{\rho(r)}{f(r)}, \quad \text { where } \quad J(r)=-\mathrm{i} \sum_{i=1}^{N(Z)} \phi_{i}^{*}(r)(\nabla \times \sigma) \phi_{i}(r) .
$$

Numerical tests of the same kind as those discussed above show that the error made in using eq. (7) is about the same as neglecting the term $\widetilde{E}_{k}^{(4)}$ in eq. (5) for the case with $f(r) \equiv 1$ and $S(r)=0$ and hence is of the order of $15-20 \mathrm{MeV}$. If higher accuracy is wanted for the kinetic energy, inclusion of the higher order terms will be necessary. The eqs. (7) and (8) may, however, be sufficient to include the effective mass and spin-orbit potential in the approach of Bohigas et al. [13]. These authors recently included the Weizsäcker term in a variational calculation using a local Skyrme force and treated the remaining error in the kinetic energy perturbatively by an exact diagonalization of the one body Hamiltonian. 
In summary we stress that the ETF approximation yields a very accurate expansion of the average kinetic energy of an independent particle system in terms of its density $\rho(r)$, if up to fourth order gradients of $\rho(r)$ are included. We have shown numerically for a local Woods-Saxon potential that the remaining error in the total average kinetic energy is only a few $\mathrm{MeV}$ and varies very little with deformation and nucleon number. The evaluation of the fourth order terms for the case of a varying effective mass and a spin-orbit potential is extremely tedious but straightforward.

We are grateful to Prof. R.K. Bhaduri for stimulating discussions and to O. Bohigas and collaborators for detailed discussions concerning ref. [13]. B.K.J. thanks the National Research Council of Canada for financial support and M.B. acknowledges the warm hospitality during a visit at the Institute of Theoretical Physics of the I.P.N. at Orsay, where some of the numerical calculations have been completed.

\section{References}

[1] D. Vautherin and D.M. Brink, Phys. Rev. C5 (1972) 626.

[2] D. Vautherin, Phys. Rev. C7 (1973) 296;

H. Flocard et al., Nucl. Phys. A203 (1973) 433;

M. Beiner et al., Nucl. Phys. A238 (1975) 29.

[3] T.H.R. Skyrme, Phil. Mag. 1 (1956) 1043; Nucl. Phys. 9 (1959) 615.

[4] J.W. Negele and D. Vautherin, Phys. Rev. C5 (1972) 1472 and C11 (1975) 1031.

[5] H. Flocard et al., Nucl. Phys. A231 (1974) 176;

M. Beiner et al., Physica Scripta 10A (1974) 84.

[6] M. Brack and P. Quantin, Phys. Lett. 56B (1975) 421.

[7] V.M. Strutinsky, Nucl. Phys. A95 (1967) 420 and A122 (1968) 1.

[8] H.A. Bethe and R.F. Bacher, Rev. Mod. Phys. 8 (1936) 82;

R.A. Berg and L. Wilets, Phys. Rev. 101 (1956) 201;

K.A. Brueckner et al., Phys. 171 (1968) 1188.

[9] D.A. Kirzhnits, Field theoretical methods in many body systems (Pergamon Press, London, 1967);

A.S. Tyapin, Sov. J. Nucl. Phys. 11 (1970) 53;

P.H.E. Gross, Phys. Lett. 42B (1972) 41 ;

C.H. Hodges, Can. J. Phys. 51 (1973) 1428;

R. Balian and C. Bloch, Ann. Phys. 63 (1971) 592;

B.K. Jennings, Ph.D. Thesis, McMaster University (1976).

[10] C.F. v. Weizsäcker, Z. Physik 96 (1935) 431.

[11] M. Brack et al., Rev. Mod. Phys. 44 (1972) 320.

[12] B.K. Jennings, R.K. Bhaduri and M. Brack, Nucl. Phys. A253 (1975) 29. 